\title{
Molecular characterization of forest soil based Paenibacillus elgii and optimization of various culture conditions for its improved antimicrobial activity
}

\author{
S. N. Kumar, Jubi Jacob, U. R. Reshma, R. O. Rajesh and B. S. D. Kumar*
}

Agroprocessing and Natural Products Division, Council of Scientific and Industrial Research - National Institute for

Interdisciplinary Science and Technology, Thiruvananthapuram, India

Microorganisms have provided a bounty of bioactive secondary metabolites with very exciting biological activities such as antibacterial, antifungal antiviral, and anticancer, etc. The present study aims at the optinuzation of culture conditions for improved antimicrobial production of Paenibacillus elgii obtained from Wayanad forest of Western Ghats region of Kerala, India. A bacterial strain isolated from the Western Ghats forest soil of Wayanad, Kerala, India was identified as $P$. elgii by 16S rRNA gene sequencing. P. elgii recorded significant board spectrum activity against all human and plant pathogenic microorganism tested except Candida albicans. It has been well

OPEN ACCESS

Edited by:

Márcia Vanusa Da Silva,

Universidade Federal

de Pernambuco, Brazil

*Correspondence:

B. S. D. Kuma

kumardileep@niist.res.in

Specialty section: This article was submitted to Antimicrobials, Resistance and Chemotherapy,

a section of the journa

Frontiers in Microbiology

Received: 05 August 2015 Accepted: 09 October 2015 Published: 21 October 2015

Citation: Kumar SN, Jacob J, Reshma UR, Rajesh RO and Kumar BSD (2015) Molecular characterization of forest soil based Paenibacillus elgii and optimization of various culture conditions for its improved antimicrobial activity.

Front. Microbiol. 6:1167. doi: 10.3389/fmicb.2015.01167 known that even minor variations in the fermentation medium may impact not only the quantity of desired bioactive metabolites but also the general metabolic profile of the producing microorganisms. Thus, further studies were carried out to assess the impact of medium components on the antimicrobial production of $P$. elgii and to optimize an ideal fermentation medium to maximize its antimicrobial production. Out of three media [nutrient/broth (NA), Luria broth (LB) and Trypticase soy broth (TSB)] used for fermentation, TSB medium recorded significant activity. Glucose and meat peptone were identified as the best carbon and nitrogen sources, which significantly affected the antibiotic production when supplemented with TSB medium. Next the effect of various fermentation conditions such as temperature, $\mathrm{pH}$, and incubation time on the production of antimicrobial compounds was studied on TSB + glucose + meat peptone and an initial $\mathrm{pH}$ of 7 and a temperature of $30^{\circ} \mathrm{C}$ for 3 days were found to be optimum for maximum antimicrobial production. The results indicate that medium composition in the fermentation media along with cultural parameters plays a vital role in the enhanced production of antimicrobial substances.

Keywords: Paenibacillus elgii, cultural condition, antimicrobial metabolites, improvement, carbon source

\section{INTRODUCTION}

During the last few decades, there has been a concomitant rise in the occurrence of multidrugresistant (MDR) pathogens that have caused serious problems to humans worldwide (Ajesh et al., 2013). The growing tendency of various microbial infections, the rapid emergence of MDR to current antibiotics and quick evolution through mutation are of a significant threat to the 
A

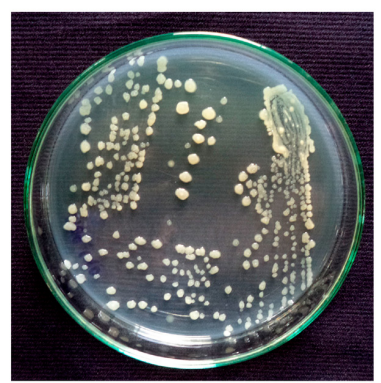

B

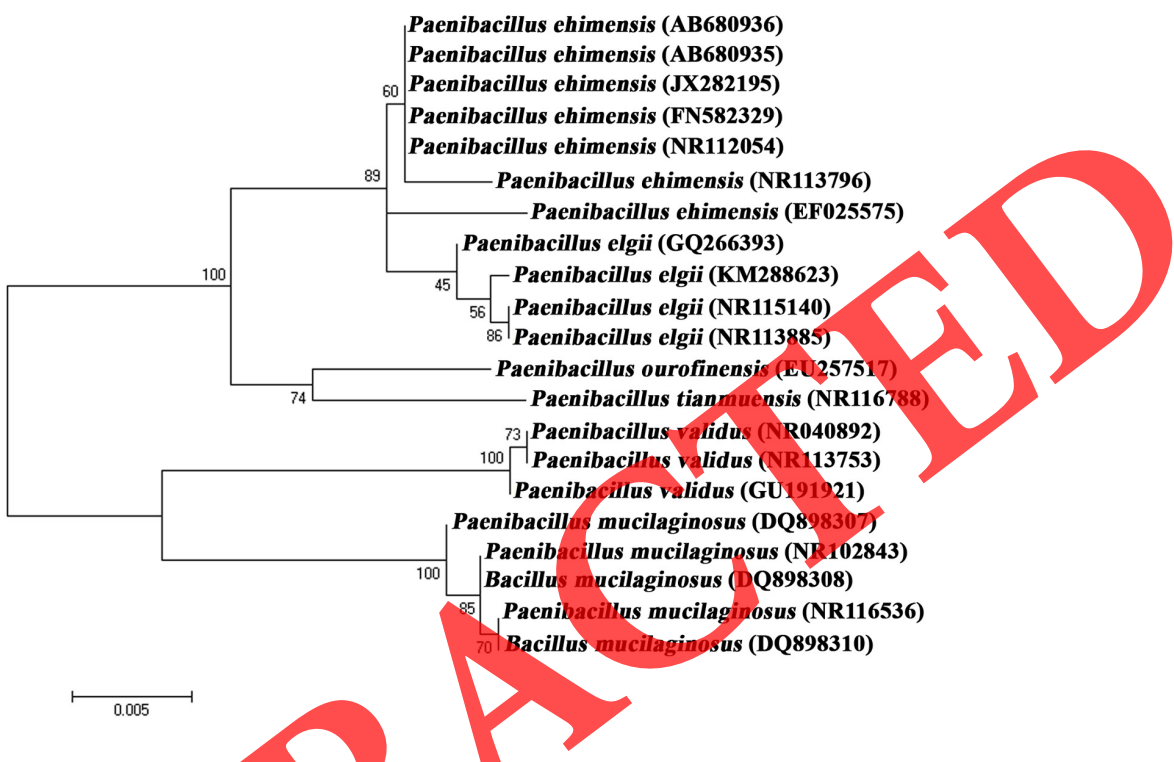

FIGURE 1 | (A) Paenibacillus elgii isolate was grown on nutrient agar plates. (B) Neighbor joining Phylogenetic tree showing the position of isolate P. elgii with other species of the genus Paenibacillus sp. and related taxabased on 16S rDNA gene sequences. Bootstrap values (expressed as percentages of 1000 replications) are indicated.

control of various microbial infections (Roy ety al., 2013). Unfortunately, few new antibiotics have been developed precisely for treating MDR microbes in recent years (Vaara et al., 2010; Velkov et al., 2010). The development of novel antibiotics cannot keep up with the evolution of antimicrobial resistance of bacteria against various drugs. Thus, there is a great need for discovering and developing new antimicrobial drugs to combat the drugresistant pathogens. The natural compounds with medicinal importance are primarily produced from the primary and secondary metabolism of various microorganisms and plants. Interestingly, microbial based natural compounds have made an incredible input to the antimicrobial drug discovery and its development process over past seven decades (Jose and Jebakumar, 2013). Microbial based natural compounds are the origin of most of the antimicrobial drugs available on the market today. But now a day there is a disturbing shortage of novel antimicrobial drugs currently under development in the pharmaceutical industry. Still, these natural compounds from microbial sources remain as the most talented source of many new antibiotics, although new tactics are still required to improve the competence of the current drug discovery process.
TABLE 1 | In vitro antibiosis of the Paenibacillus elgii against test microbes.

\begin{tabular}{lccc}
\hline Test bacteria & $\begin{array}{c}\text { Zone of } \\
\text { Inhibition } \mathbf{( m m )}\end{array}$ & Test fungi & $\begin{array}{c}\text { Zone of } \\
\text { Inhibition (mm) }\end{array}$ \\
\hline B. cereus & $55 \pm 3.25$ & A. flavus & $17 \pm 1.27$ \\
B. subtilis & $27 \pm 2.15$ & C. albicans & - \\
S. aureus & $41 \pm 1.75$ & T. rubrum & $25 \pm 0$ \\
S. epidermis & $52 \pm 2.75$ & A. niger & $22 \pm 0.57$ \\
S. simulans & $47 \pm 2.12$ & A. fumigatus & $29 \pm 1.52$ \\
E. coli & $48 \pm 3.15$ & A. tubengensis & $20 \pm 0.77$ \\
K. pneumoniae & $40 \pm 1.85$ & F. oxysporum & $15 \pm 0.52$ \\
P. mirabilis & $25 \pm 0$ & R. solani & $14 \pm 1.77$ \\
P. aeruginosa & $44 \pm 1$ & C. gloeosporioides & $17 \pm 0.77$ \\
S. typhi & $35 \pm 1.85$ & P. expansum & $31 \pm 1$ \\
\hline
\end{tabular}

-, Recorded no activity.

Production of bioactive secondary metabolites by microorganisms significantly depends on the species and strains of microorganisms, its nutritional, and cultural conditions (Jose et al., 2011; Wang et al., 2011). Minor variations in 


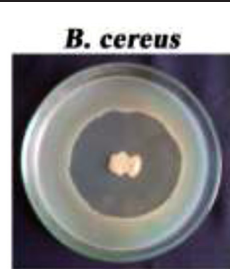

E. coli

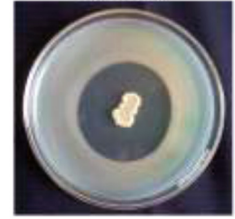

B. subtilis

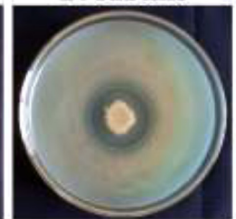

K. pneumoniae

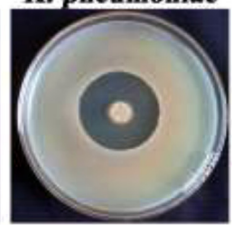

S. aureus

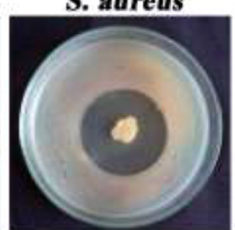

P. mirabilis

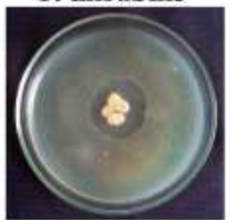

S. epidermis

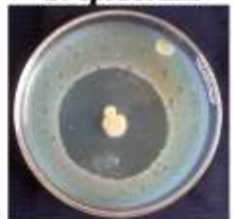

P. aeruginosa

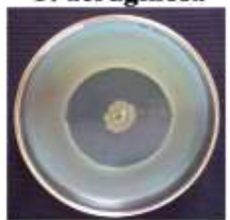

S. simulans

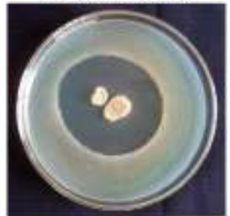

S. typhi

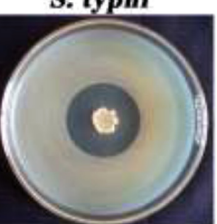

FIGURE 2 | In vitro antibiosis of $P$. elgii against test bacteria.

A

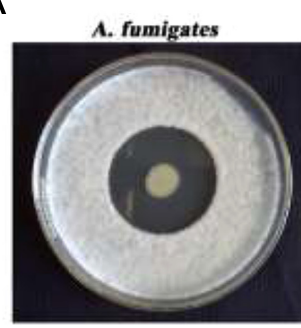

A. tubengensis

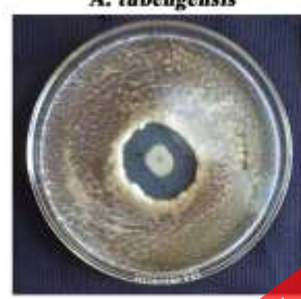

A. niger

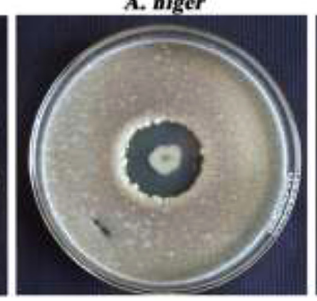

P. expansum

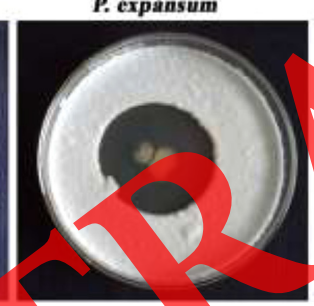

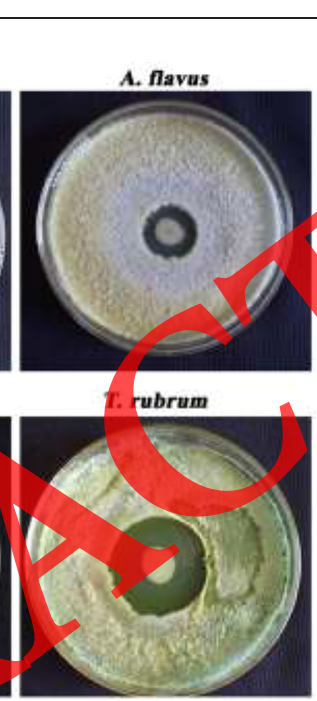

B

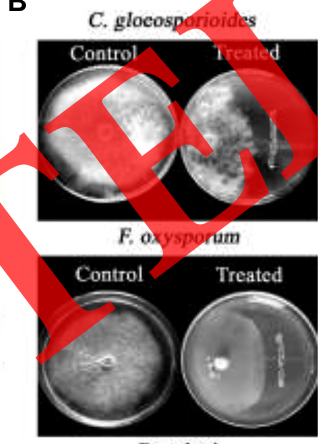

R. solani

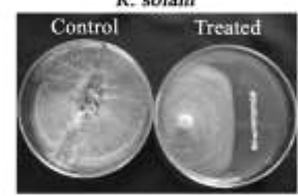

FIGURE 3 | In vitro antibiosis of $P$. elgii against test fungi. (A) Sporulation fungi and (B) filamentous fungi.

the fermentation media composition play a profound role in quantity and quality of secondary metabolites production (Wang et al., 2011). Hence, optimization of the fermentation medium is very much essential to confirm enhanced production of desired metabolites. Improving the antibiotic production is the prerequisite to achieving adequate antibiotic yield for evaluating its potential and novelty. The antibiotic producing ability of the microorganism is not a static property and it is highly influenced by culture conditions and media components used (Hassan et al., 2001; Gunnarsson et al., 2003). Therefore, designing a suitable fermentation medium and conditions has vital importance in improving the antibiotic yield and easing the cost of production (Wang et al., 2008; Feng et al., 2011). Moreover designing an appropriate fermentation system for a good producer strains require a series of trials like selection of basal medium, selection of best carbon and nitrogen sources, optimization of the physical parameters, screening for each medium components which have significant impact on production of particular compound, and optimization of influencing medium components (Wang et al., 2011). However, studies on the development of ideal production medium and culture conditions for the consistent production of antibiotics from Paenibacillus elgii remain scarce in the literature. Thus, the present paper is the first report on the optimization of the culture conditions for the maximum production of antimicrobial metabolites by $P$. elgii.

\section{MATERIALS AND METHODS}

\section{Strain and Its Maintenance}

The Paenibacillus strain used in this work designated as P. elgii NIISTB523 was isolated from the forest soil of Western Ghats region of Wayanad, Kerala, India and has the ability of producing antimicrobial metabolites against a wide range of bacteria. Briefly, soil samples (approximately $10 \mathrm{~g}$ ) were collected in a sterile polythene bag using a sterile spatula. $1 \mathrm{~g}$ of the soil samples were dissolved in $10 \mathrm{ml}$ of sterile distilled water to make soil suspensions. Portions of the suspension were inoculated on the 
TABLE 2 | Antimicrobial activity of crude extract.

\begin{tabular}{lccc}
\hline Test microbes & \multicolumn{3}{c}{ Zone of inhibition $(\mathbf{m m})$} \\
\cline { 2 - 4 } & TSB & LB & NB \\
\hline B. cereus & $27 \pm 0$ & $21 \pm 0$ & $11 \pm 0$ \\
B. subtilis & $23 \pm 1.15$ & $16 \pm 1$ & $13 \pm 0$ \\
S. aureus & $20 \pm 1.72$ & $17 \pm 1.12$ & - \\
S. epidermis & $25 \pm 0.52$ & $15 \pm 1.77$ & $12 \pm 0$ \\
S. simulans & $23 \pm 1$ & $20 \pm 1.12$ & $15 \pm 1$ \\
E. coli & $21 \pm 1$ & $12 \pm 0$ & $13 \pm 1.72$ \\
K. pneumoniae & $24 \pm 0$ & $14 \pm 1.75$ & $12 \pm 2.20$ \\
P. mirabilis & $20 \pm 2.25$ & $13 \pm 0$ & - \\
P. aeruginosa & $21 \pm 1.75$ & $11 \pm 1.12$ & - \\
S. typhi & $24 \pm 1.12$ & $17 \pm 1$ & - \\
\hline
\end{tabular}

-, Recorded no activity

nutrient agar and incubated at $37^{\circ} \mathrm{C}$ for $24 \mathrm{~h}$. After which P. elgii colonies showing a clear zone of inhibition were isolated. After isolation, the $P$. elgii was maintained on nutrient agar (NA) medium at $30^{\circ} \mathrm{C}$.

\section{Molecular Identification of the Organism}

Genomic DNA was isolated from the bacterium using DNeasy ${ }^{\circledR}$ Blood and Tissue Kit (Qiagen) following manufacturer's instructions. A portion of the culture was transferred to $180 \mu \mathrm{l}$ of ATL buffer and followed by the addition of proteinase $\mathrm{K}$ $(20 \mu \mathrm{l})$ and incubated at $56^{\circ} \mathrm{C}$ in a water bath until the cells were fully lysed. After lysis, RNase A (5 $\mu \mathrm{l}, 100 \mathrm{mg} / \mathrm{ml})$ was added and incubated for $5 \mathrm{~min}$ at room temperature. Then $200 \mu \mathrm{l}$ of AL buffer and $200 \mu \mathrm{l}$ of ethanol (100\%) were added followed by thorough mixing by vortexing. After that, the mixture was transferred into DNeasy Mini spin column placed in a $2 \mathrm{ml}$ collection tube and centrifuged at $8000 \mathrm{rpm}$ for $1 \mathrm{~min}$. After centrifugation the DNeasy mini spin column was shifted to a new $2 \mathrm{ml}$ tube and washed with $500 \mathrm{pl}$ of the AW 1 buffer. Wash step was repeated using the AW2 buffer. After through washing the DNeasy mini spin column was placed in a clean $1.5 \mathrm{ml}$ tube and DNA was eluted out using $50 \mu \mathrm{l}$ of AE buffer.

The quality of the isolated DNA was checked using agarose gel electrophoresis. The complete 16S rRNA fragment was prepared by PCR and the amplification reactions were carried out in a $20 \mu \mathrm{l}$ reaction volume which contained $1 \mathrm{X}$ PCR buffer (100 mM Tris-HCl, pH 8.3; $500 \mathrm{mM} \mathrm{KCl}$ ), $0.2 \mathrm{mM}$ each dNTPs (dATP, dGTP, dCTP, and dTTP), $2.5 \mathrm{mM}$ $\mathrm{MgCl}_{2}, 1$ unit of AmpliTaq Gold DNA polymerase enzyme, $0.1 \mathrm{mg} / \mathrm{ml} \mathrm{BSA}, 4 \% \mathrm{DMSO}, 5 \mathrm{pM}$ of forward (16S-UP-FCGAATTCGTCGACAACAGAGTTTGATCCTGGCTCAG) and reverse (16S-UP-R-CCCGGGATCCAAGCTTACGGCTA CCTTGTTACGACTT) primers and FTA disk as template. The PCR amplification was carried out in a PCR thermal cycler (GeneAmp PCR System 9700, Applied Biosystems, USA). PCR conditions consisted of an initial denaturation step at $94^{\circ} \mathrm{C}$ for $5 \mathrm{~min}$ followed by 35 amplification cycles of $94^{\circ} \mathrm{C}$ for $1 \mathrm{~min}$, $55^{\circ} \mathrm{C}$ for $45 \mathrm{~s}$, and $72^{\circ} \mathrm{C}$ for $1 \mathrm{~min}$. Sequencing reaction was done in a PCR thermal cycler (Gene Amp PCR System 9700,

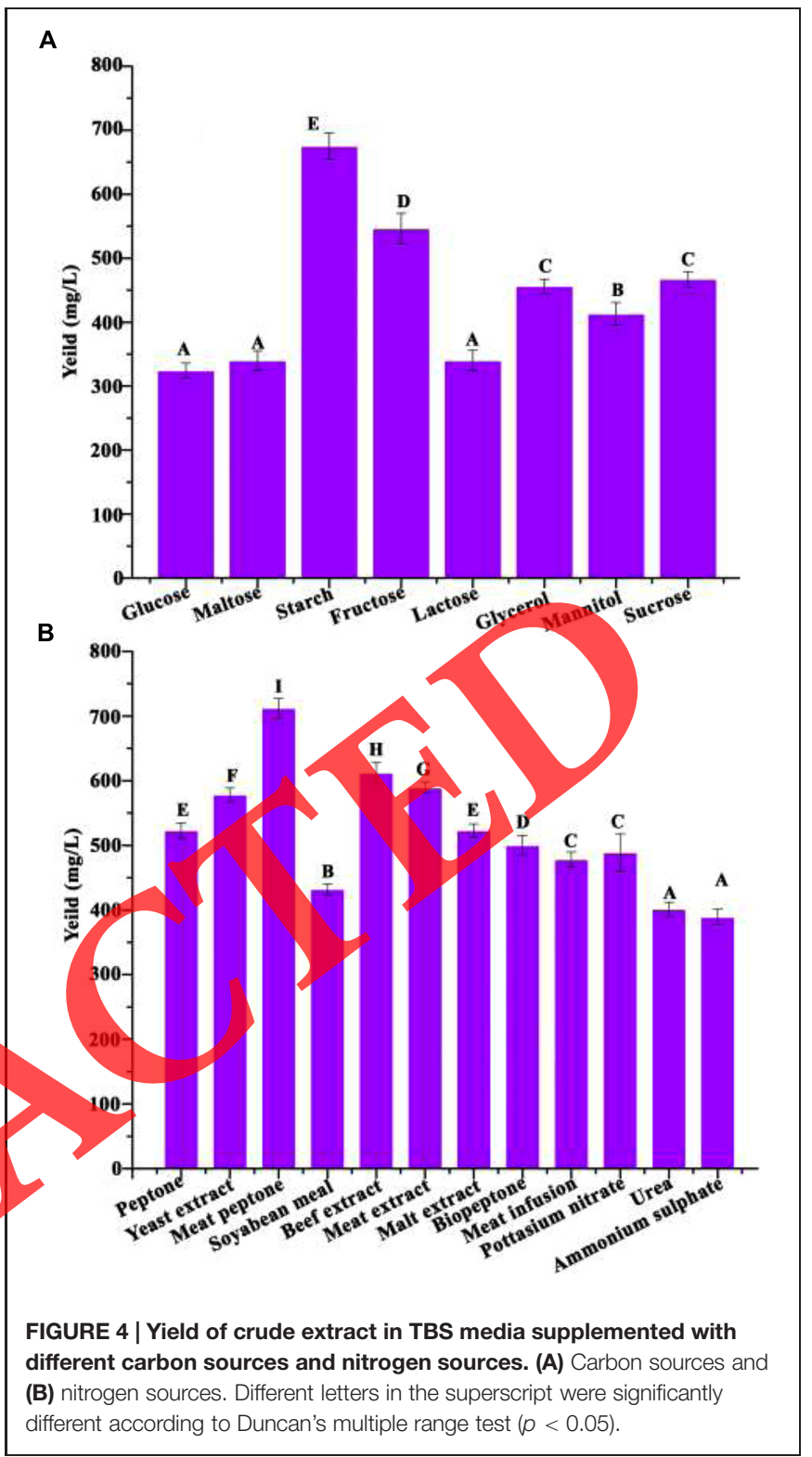

Applied Biosystems, USA) using the Big Dye Terminator v3.1 Cycle Sequencing Kit (Applied Biosystems, USA) following manufacturers protocol. The sequence quality was checked using Sequence Scanner Software v1 (Applied Biosystems). Sequence alignment and required editing of the obtained sequences were carried out using Geneious Pro v5.6 (Drummond et al., 2012).

\section{Phylogenetic Analysis}

The nucleotide sequence obtained was finally processed to remove low-quality reads, transformed into consensus sequences with Geneious Pro software version 5.6. The resulted high-quality sequences were analyzed with BLASTn (NCBI) to confirm the final authenticity of the isolated bacterium. The DNA sequences of closely related species and genus were downloaded from the NCBI Genbank database and phylogenetic conductions was carried out with the MEGA version 5 program. Then 
TABLE 3 | Antimicrobial activity of crude extract in TBS media supplemented with different carbon sources.

\begin{tabular}{|c|c|c|c|c|c|c|c|c|}
\hline \multirow[t]{2}{*}{ Test microbes } & \multicolumn{8}{|c|}{ Zone of inhibition (mm) } \\
\hline & Sta & Mal & Glu & Fru & Lac & Gly & Man & Suc \\
\hline B. subtilis & $15 \pm 1$ & $13 \pm 1.12$ & $27 \pm 0$ & $22 \pm 1.11$ & $11 \pm 0.52$ & $21 \pm 0.52$ & $12 \pm 0.57$ & $22 \pm 1.12$ \\
\hline S. aureus & $16 \pm 1$ & $19 \pm 052$ & $23 \pm 0$ & $16 \pm 0.77$ & $14 \pm 0$ & $22 \pm 0$ & $14 \pm 0$ & $21 \pm 0$ \\
\hline S. epidermis & $14 \pm 0$ & $15 \pm 1$ & $28 \pm 1$ & $23 \pm 1.12$ & $15 \pm 1.12$ & $24 \pm 1$ & $13 \pm 0$ & $19 \pm 1.56$ \\
\hline S. simulans & $16 \pm 1.12$ & $21 \pm 1$ & $27 \pm 1.15$ & $23 \pm 1.57$ & $13 \pm 0.77$ & $25 \pm 1.27$ & $13 \pm 1$ & $20 \pm 0$ \\
\hline B. cereus & $19 \pm 0.77$ & $22 \pm 0$ & $29 \pm 0.52$ & $21 \pm 2.17$ & $17 \pm 1$ & $21 \pm 1.56$ & $12 \pm 1$ & $18 \pm 1$ \\
\hline E. coli & $20 \pm 2.21$ & $14 \pm 0$ & $26 \pm 0.77$ & $25 \pm 2.72$ & $19 \pm 1$ & $26 \pm 2.72$ & $16 \pm 1.12$ & $17 \pm 1.72$ \\
\hline K. pneumoniae & $21 \pm 0$ & $19 \pm 1.12$ & $31 \pm 1.17$ & $21 \pm 1.77$ & $21 \pm 0$ & $19 \pm 1.77$ & $15 \pm 0.57$ & $15 \pm 1.77$ \\
\hline P. mirabilis & $23 \pm 1$ & $23 \pm 1.77$ & $26 \pm 1.72$ & $17 \pm 0.52$ & $14 \pm 1.12$ & $18 \pm 1.52$ & $19 \pm 0.52$ & $19 \pm 1.52$ \\
\hline$P$. aeruginosa & $18 \pm 0.77$ & $21 \pm 1.52$ & $24 \pm 0.77$ & $18 \pm 0$ & $17 \pm 1.77$ & $23 \pm 0.57$ & $21 \pm 1.52$ & $21 \pm 0.57$ \\
\hline S. typhi & $19 \pm 0.52$ & $22 \pm 2.12$ & $25 \pm 0.52$ & $19 \pm 1$ & $15 \pm 0.22$ & $21 \pm 0.52$ & $20 \pm 1.77$ & $18 \pm 2.12$ \\
\hline
\end{tabular}

Bold indicated the best activity displayed by glucose against K. pneumoniae.

the sequences were aligned using the ClustalW and were then analyzed to determine the relationships between other closely related isolates by the neighbor-joining method using the Maximum Composite Likelihood model. Bootstrap values were produced using 1500 replicates.

\section{Test Pathogens}

\section{Bacteria}

Gram-positive bacteria: Bacillus subtilis MTCC 2756, Staphylococcus aureus MTCC 902, S. epidermidis MTCC 435 and S. simulans MTCC 3610; Gram-negative bacteria: Escherichia coli MTCC 2622, Klebsiella pneumoniae MTCC 109, Proteus mirabilis MTCC 425, Vibrio cholerae MTCC 3905, Pseudomonas aeruginosa MTCC 2642, and Salmonella typhi MTCC 3216.

\section{Fungi}

Medically important fungi: Aspergillus flavus MTCE 183, and Trichophyton rubrum MTCC 296, Candida albicans MTCC 277 and agriculturally important fungi: A. niger MTCC 282, A. fumigatus MTCC 3376, A tubingensis MTCC 2425, Fusarium oxysporum MTCC 284, Rhizoctonia solani MTCC 4634, Colletotrichum gloeosporioides MTCC 10183, and Penicillium expansum MTCC 2006. All the test microorganisms were procured from Microbial Type Culture Collection Centre (MTCC), IMTECH, Chandigarh, India. The test bacteria were maintained on nutrient agar slants and test fungi were maintained on potato dextrose agar slants.

\section{In Vitro Antibiosis of $P_{\text {. elgii }}$ Against Bacteria}

The agar overlay method was used for the examination of intact $P$. elgii inhibitory effect against test bacterial pathogens. In this method, the plate containing the 2 days old culture of $P$. elgii strain was overlaid with the top nutrient agar (Hi-media, Mumbai, India) which contains the test bacterial strains. The inhibitory effect was evaluated after $18 \mathrm{~h}$ incubation at $37^{\circ} \mathrm{C}$ and the results were evaluated by the measurement of inhibition zone of diameter expressed in millimeter $(\mathrm{mm})$.

\section{Against Fungi}

The dual culture technique was employed to find out the inhibitory activity of $P$. elgit against filamentous fungi (F. oxysporum, R. solani, and C. gloeosporioides) on PDA agar medium (Naing et al., 2014). The dual cultured plates after treatment were then incubated for 5 days at $28^{\circ} \mathrm{C}$. The agar overlay method with fungal spores was employed for detecting the actiyity of $P$. eligii against spore forming test fungi. P. elgii were inoculated as spot on mutrient agar plates and incubated at $30^{\circ} \mathrm{C}$ for $48 \mathrm{~h}$. Then the plates were overlaid with potato dextrose agar containing $1 \times 10^{6}$ spores of each fungus per ml. After $48-72 \mathrm{~h}$ of incubation at $30^{\circ} \mathrm{C}$, the inhibition zone was measured. For Candida sp. agar overlay method, as described above for the bacteria, were adopted.

\section{Standardization of Optimal Fermentation Medium for Maximum Antimicrobial Production}

Three different standard media such as nutrient broth (NA), Luria broth (LB), and Trypticase soy broth (TSB) were used in comparative studies to find the best fermentation medium for antimicrobial production. Five microliter of the seed culture was transferred into $100 \mathrm{ml}$ of different sterile medium in $250 \mathrm{ml}$ flask. The flasks were incubated in the dark at $150 \mathrm{rpm}$ for $72 \mathrm{~h}$ at $30^{\circ} \mathrm{C}$ on a rotary shaker. After incubation, the culture media were then centrifuged $\left(10,000 \mathrm{~g}, 20 \mathrm{~min}, 4^{\circ} \mathrm{C}\right)$ followed by filtration through a $0.45 \mu \mathrm{m}$ filter, to obtain cell free culture filtrate.

\section{Preparation of Crude Organic Extract}

The cell free culture filtrate was neutralized with concentrated $\mathrm{HCl}$ and extracted with an equal volume of ethyl acetate thrice. The ethyl acetate extracts were combined, dried over anhydrous sodium sulfate, and concentrated using a rotary flash evaporator at $40^{\circ} \mathrm{C}$ to obtain the crude extract.

\section{Assay of Antibiotic Activity of Crude Extract (Agar Disk Diffusion Assay)}

Antibiotic activity of the crude extracts was measured by agar disk diffusion assay. Briefly, $0.1 \mathrm{ml}$ containing $10^{6}-10^{7} \mathrm{CFU} / \mathrm{ml}$ 


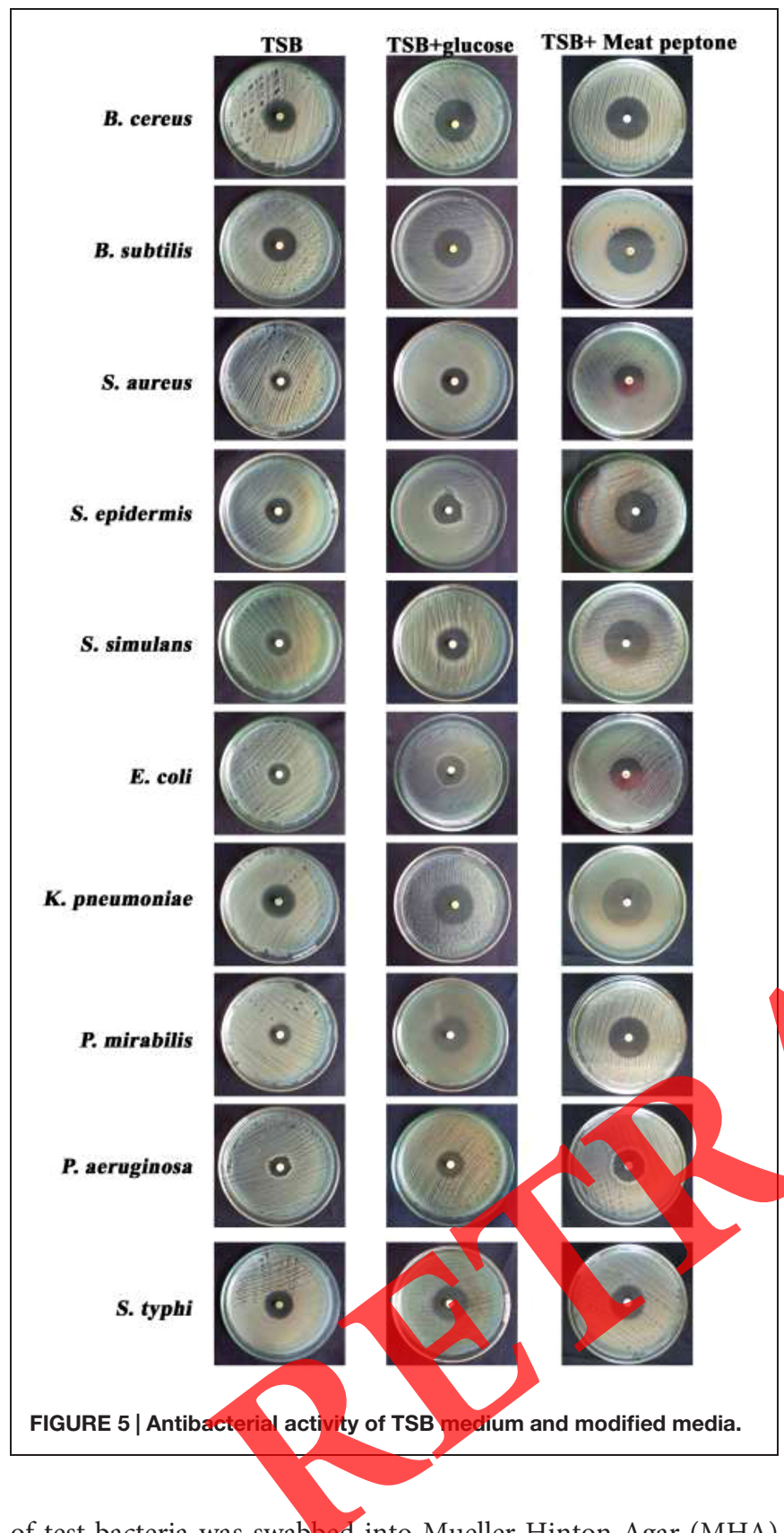

of test bacteria was swabbed into Mueller Hinton Agar (MHA) plate. The crude extract $(100 \mu \mathrm{l})$ following microfiltration using a $0.22 \mu \mathrm{m}$ syringe microfilter, were loaded on $6 \mathrm{~mm}$ sterile disk filters (Whatman 3-mm paper, Hi-media) and air dried. After $2 \mathrm{~h}$ incubation at room temperature, the dried disks were placed on the MHA plate and incubated at $37^{\circ} \mathrm{C}$ for $18 \mathrm{~h}$ to determine the diameter of zone of inhibition and expressed in millimeter $(\mathrm{mm}$; Wang et al., 2011). The experiment was performed in triplicate.

\section{Selection of Best Carbon and Nitrogen Sources for Maximum Antimicrobial Production}

In order to examine the effect of the different carbon and nitrogen sources on the antimicrobial production of the potential microbe, the best nutrient medium for antimicrobial production was employed as an original medium for the following optimization studies. In this study, TSB media was used as original medium (recorded maximum activity in the initial standardization studies). One percentage (1\%) of eight simple and complex carbon (glucose, maltose, starch, fructose, lactose, glycerol, mannitol, and sucrose) and 12 nitrogen (peptone, yeast extract, meat peptone, soyabean meal, beef extract, meat extract, malt extract, biopeptone, meat infusion, potassium nitrate, urea, ammonium sulfate) sources were used independently instead of the corresponding carbon and nitrogen sources in the original best nutrient medium while other medium components were kept constant at its original concentration. The bacterial fermentation was carried out for $72 \mathrm{~h}$ of incubation at $28^{\circ} \mathrm{C}$ with shaking at $150 \mathrm{rpm}$ and the crude extract was isolated according to the method mentioned earlier. The antimicrobial activity of the crude extract was determined by agar disk diffusion assay.

\section{Impact of Temperature, $\mathrm{pH}$ and Incubation Period on the Enhanced Production of Antimicrobial Compounds}

The outcome of eulture condition on the production of the enhanced antimicrobial production was studied on TSB supplemented with $1 \%$ of glucose and meat peptone against K. preumoniae (recorded significant activity in initial standardization studies). The impact of temperature on growth and production of antimicrobial compounds was investigated on TSB + glucose + meat peptone at different temperatures $\left(20,22,25,28,30,32,35,38\right.$, and $\left.40^{\circ} \mathrm{C}\right)$ at $\mathrm{pH}$. Ten $\mathrm{ml}$ suspension of the bacterial isolate was inoculated into $100 \mathrm{ml}$ of TSB + glucose + meat peptone in $250 \mathrm{ml}$ Erlenmeyer flask and incubated in a shaking incubator upheld at $150 \mathrm{rpm}$ for 4 days at $28^{\circ} \mathrm{C}$. The antimicrobial activity was assessed against K. pneumoniae by agar disk diffusion assay as mentioned above. Likewise, the impact of $\mathrm{pH}$ on the antimicrobial compounds production was studied at various $\mathrm{pH}$ range (4-9) using $\mathrm{TSB}+$ glucose + meat peptone by incubating at $28^{\circ} \mathrm{C}$ for 4 days. The impact of incubation period on the antimicrobial production was also studied in the similar way as above by incubating for various days $(1-7)$ at $28^{\circ} \mathrm{C}$ using TSB + glucose + meat peptone at $\mathrm{pH} 7$.

\section{Antibacterial Activity of TSB + Glucose + Meat Peptone in the Best Ideal Condition}

$\mathrm{TSB}+$ glucose + meat peptone with an initial $\mathrm{pH}$ of 7 was prepared and the fermentation was carried for 3 days at $30^{\circ} \mathrm{C}$ (best ideal condition that was already standardized) under shaking $(150 \mathrm{rpm})$. The crude extract was isolated after fermentation as described earlier and the antibacterial activity was carried out using agar disk diffusion assay against all test bacteria. Ciprofloxacin was used as reference antibiotic.

\section{Statistical Analysis}

The statistical analyses of the data were achieved using SPSS (Version 17.0; SPSS, Inc., Chicago, IL, USA). Antimicrobial 


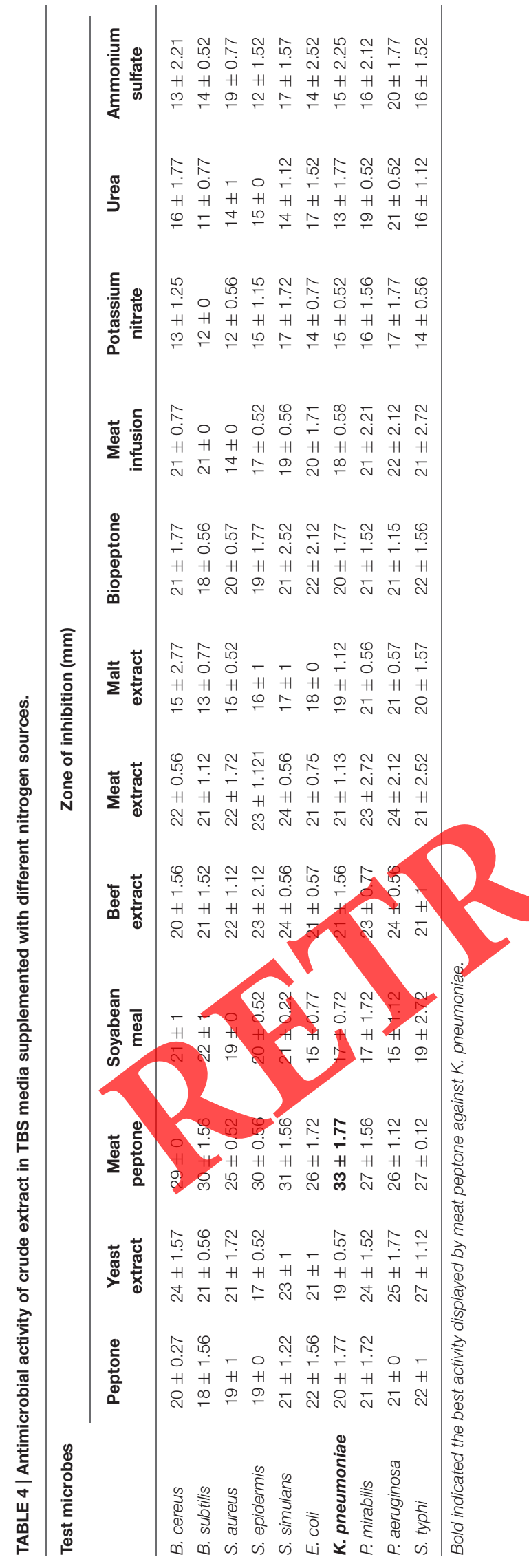

results were reported as mean \pm standard deviations. Duncans multiple range tests (DMRT) was done to compare that the sample means were significantly different from each other at a significant level of $P>0.05$.

\section{Results}

\section{S rRNA Sequencing and Phylogenetic Analysis of the Strain}

The isolate NIISTB523 was identified as P. elgii based on $16 \mathrm{~S}$ rRNA gene sequencing. PCR amplification yielded $\sim 1500 \mathrm{bp}$ amplicon. Blast analysis showed greater than $99 \%$ similarity to $P$. elgii sequences available in the NCBI Genbank database and thus the isolates were finally acknowledged as $P$. elgii.

Sequence data for the 16S rRNA gene of $P$. elgii was finally deposited in the NCBI GenBank nucleotide database under the Ac. No. (KM288623). The phylogenetic tree clearly showed the relationships of the isolates used in the analysis. The present bacterium ( $P$. elgii) was successfully grouped along with other $P$. elgii isolates obtained from the NCBI Genbank database confirming the genumeness of the isolate (Figure 1).

\section{In Vitro Antibiosis of the P. elgii Against the Test Microbes}

In vitro antibiosis of the P. elgii against the test bacteria was shown in Table 1. P. elgii displayed significant antimicrobial activity against all test bacteria and the best activity was recorded against B. cereus $(55 \mathrm{~mm})$ and S. epidermidis $(52 \mathrm{~mm}$; Table 1 and Figure 2). P. elgii also recorded significant in vitro antifungal activity against all the test fungi except $C$. albicans and significant aetivity was recorded against $P$. expansum $(31 \mathrm{~mm}$; Table 1 and Figure 3).

\section{Standardization of Optimal Fermentation Medium for Maximum Antimicrobial Production}

\section{Yield of Crude Extract in Standard Media}

The maximum yield was recorded for TSB $(567 \mathrm{mg} / \mathrm{L})$, followed by $\mathrm{LB}(423 \mathrm{mg} / \mathrm{L})$, and nutrient broth $(235 \mathrm{mg} / \mathrm{L})$.

\section{Antibacterial Activity of Crude Extract}

The antibacterial activity of crude extracts from standard media is shown in Table 2. TSB medium recorded maximum activity. The most sensitive test bacteria to the TSB crude extract was B. cereus $(27 \mathrm{~mm})$.

\section{Selection of Best Carbon and Nitrogen Sources for Maximum Antimicrobial Production}

The yield of crude extract from TSB supplemented with different carbon and nitrogen sources are shown in Figure 4. In case of TSB supplemented with different carbon sources, the highest yield was recorded by TSB + glucose, followed by TSB + fructose (Figure 4A). Whereas in the case of TSB supplemented with 


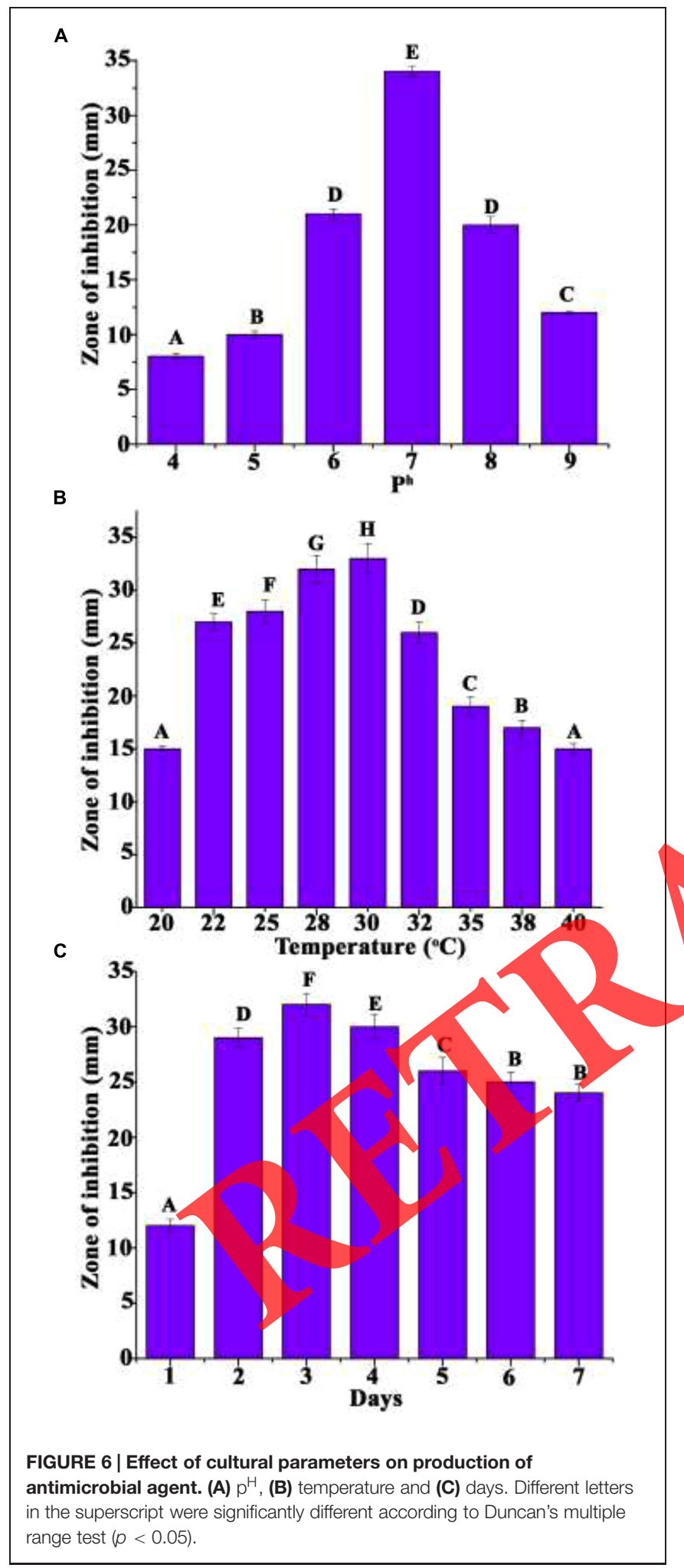

different nitrogen sources, the highest yield was recorded by $\mathrm{TSB}+$ meat peptone (Figure 4B).

In case of the antimicrobial activity of crude extract from different carbon sources, significant activity was recorded
TABLE 5 | Antibacterial activity of crude extract from TSB + Glucose + meat peptone in the best ideal condition.

\begin{tabular}{lcc}
\hline Test microbes & \multicolumn{2}{c}{ Zone of inhibition (mm) } \\
\cline { 2 - 3 } & Extract & Ciprofloxacin \\
\hline B. cereus & $29 \pm 0.52$ & $31 \pm 0$ \\
B. subtilis & $30 \pm 1.12$ & $33 \pm 1.15$ \\
S. aureus & $32 \pm 0.77$ & $29 \pm 1.72$ \\
S. epidermis & $31 \pm 1.24$ & $28 \pm 1.52$ \\
S. simulans & $28 \pm 1$ & $27 \pm 1.52$ \\
E. coli & $33 \pm 0.57$ & $29 \pm 0$ \\
K. pneumoniae & $36 \pm 1.52$ & $30 \pm 1.75$ \\
P. mirabilis & $32 \pm 0.77$ & $31 \pm 0$ \\
P. aeruginosa & $29 \pm 1.72$ & $32 \pm 1.12$ \\
S. typhi & $34 \pm 1.72$ & $31 \pm 1.12$ \\
\hline
\end{tabular}

by TSB + glucose (Table 3 and Figure 5), followed by TSB + glycerol. Similarly in case of TSB supplied with nitrogen sources, significant activity was recorded by TSB + meat peptone (Table 4 and Figure 5), followed TSB + yeast extract. $K$. pneumoniae was the most sensitive organism to the extract (Tables 3 and 4). All the combination recorded antimicrobial activity. But certain combination recorded lower activity than the TSB mediumalone.

\section{Effect of Various Cultural Parameters on} Antimicrobial Production

The various environmental parameters and cultural conditions for the production of the antimicrobial compounds by P. elgii have been studied on TSB + glucose + meat peptone in shaking flask condition. The present strain $P$. elgii recorded a narrow range of incubation temperature for relatively good growth and antimicrobial production. The temperature in the range of $28-30^{\circ} \mathrm{C}$ was found to be ideal for maximum antimicrobial compound production by $P$. elgii (Figure 6A). Antimicrobial activity was considerably reduced after $35^{\circ} \mathrm{C}$. The best growth, as well as maximum antimicrobial compound production, was obtained at $\mathrm{pH} 7$ (Figure 6B). Incubation period up to 7 days was found to be optimum for maximum growth as well as antimicrobial agent production by $P$. elgii (Figure 6C).

\section{Antibacterial Activity of}

\section{TSB + Glucose + Meat Peptone in the Best Ideal Condition}

Table 5 showed the antibacterial activity of the crude extract of TSB + Glucose + meat peptone against test bacteria. $K$. pneumonia exhibited significant activity with a diameter of zone of inhibition of $36 \mathrm{~mm}$.

\section{DISCUSSION}

For several years, actinomycetes have been productive sources of novel antimicrobial molecules (Nguyen et al., 2008; Ridley et al., 2008). Only recently, bacteria from other taxonomic groups 
arise as a practical novel source for various bioactive compounds with antimicrobial property and numerous successful efforts have been made in this field (Zhang et al., 2006; Wright and Sutherland, 2007; Blunt et al., 2009). In the present study, new soil bacterium exhibiting broad spectrum antimicrobial activity against various pathogens was subjected to $16 \mathrm{~S}$ rRNA sequence screening to include the well-documented antimicrobial producers and identified as P. elgii.

The genus Paenibacillus was well-defined in 1993 after an extensive comparative investigation of $16 \mathrm{~S}$ rRNA gene sequences of more than 51 species of the genus Bacillus (Ash et al., 1993). One of the enthralling characteristics of Paenibacilli is that some species of this genus are Gramnegative or Gram-variable although the Gram-positive dominate, consistent with their Bacillus originality (Elo et al., 2001; Rodriguez-Diaz et al., 2005; Roux et al., 2008). Diverse Paenibacillus species are well found in soil and rhizosphere region of plants. Several Paenibacillus species have been reported as potential biological control agents as they can produce a wide range of potent antimicrobial compounds. For example, Paenibacillus polymyxa E681, a plant growthpromoting (PGPR) rhizobacterium, which can successfully control the pre-emergence and post-emergence damping off diseases on sesame plants (Ryu et al., 2006). Production of antimicrobial compound has been considered as one of the chief mechanisms of plant diseases control with living microorganisms. So far, several Paenibacillus species have been reported to produce many antimicrobial agents with a wide spectrum inhibition of various human pathogenic bacteria and fungi (Li et al., 2007). In the present study, P. elgii displayed inhibitory activity against a number of Gram-positive and negative bacteria and also recorded significant antifungal activity against medically and agriculturally important fungi except C. albicans. C. albicans usually grow in biofilm matrix linked to $\beta-1,3$ glucan and exhibit a $\beta-1,3$ glucan matrix associated đrug resistance mechanism against various antifungal drugs (Mitchell et al., 2013). This may be the reason for the absence of activity by P. elgii against C. albicans.

The investigation on the enhanced production of antimicrobial compounds usually involves an exploration for a appropriate fermentation medium. Fermentation media were reported to have a great influence on antimicrobial metabolite production by microorganisms (Vijayakumari et al., 2013). In our study, out of three standard fermentation media used, TSB medium recorded significant antimicrobial activity. The results of the present study clearly indicated that medium composition play a significant role in the antimicrobial secondary metabolite production by bacteria. Carbon and nitrogen sources in the fermentation media were reported to have a profound influence on the production of secondary metabolite by the microorganism (Narayana and Vijayalakshmi, 2008; Kumar et al., 2012). During our fermentation studies with different carbon sources, glucose was easily assimilated and resulted in a rapid growth for $P$. elgii. The results agreed with other Paenibacillus including Paenibacillus polymyxa RNC-D (Serrano et al., 2012). Nevertheless, glucose was reported to be a repressor of secondary metabolisms (Demain,
1999). This may be because most of the carbons were utilized rapidly for the synthesis of cellular material and little would be available for antibiotic synthesis (Pandey et al., 2005). But Kumar et al. (2013) reported fructose as the best carbon source among the water-soluble carbon sources like glucose, maltose, glycerol, mannitol, and sucrose for antimicrobial metabolite production by a Bacillus sp. Considering the various carbon source, simple carbon sources such as glucose, fructose, and sucrose can enhanced growth as well as bioactive metabolite production rather than more complex carbons (Calvo et al., 2002). The nature of the nitrogen source used has a notable effect on the production of the antimicrobial metabolite by the bacterium. In our study yeast extract recorded the significant effect on the antimicrobial production followed by meat peptone and beef extract. Depending on the biosynthetic pathways involved, nitrogen sources may significantly affect antibiotic formation (Gesheva et al., 2005). The results of the present study showed the requirement of the antimicrobial agent synthesis on the various medium constituents. In fact, it has been also recorded that the nature of carbon as well as nitrogen sources strongly affects antibiotic production by various microorganisms (Vilches et al.,) 1990). It was clear from the present study that the production of antimicrobial agents by $P$. elgii was absolutely affected by the nature and type of carbon and nitrogen sources used in the fermentation medium. The present results also recorded that antimicrobial compound production was higher in fermentation medium having glucose and meat peptone as carbon and nitrogen source, Environmental factors such as $\mathrm{pH}$ and temperature are cknowledged to have a thoughtful influence on growth and antibiotic production in various bacterial species (Vijayakumari al., 2013). The changes in external $\mathrm{pH}$ of fermentation medium affect several cellular processes of microorganism such as regulation and biosynthesis of secondary metabolites (Sole et al., 1997). The $\mathrm{pH}$ of 6.5-7.5 and temperature of $25-30^{\circ} \mathrm{C}$ was reported to be the optimum for antibiotic production by Bacillus sp. (Vijayakumari et al., 2013). The $P$. elgii showed a narrow range of $\mathrm{pH} 7$ for relatively good growth and antimicrobial agent production. The maximum growth, as well as highest antimicrobial activity by $P$. elgii, was achieved at $\mathrm{pH} 7$ during the production of antimicrobial agent drastically reduced before and after $\mathrm{pH} 6$ and 8 . There are reports concerning the role of medium $\mathrm{pH}$ in the production of the bioactive metabolite especially antibiotics by microorganisms (Guimaraes et al., 2004). The P. elgii presented a narrow range of incubation temperature for comparatively good growth and antimicrobial compound production. Maximum antimicrobial compound production was obtained at $28-30^{\circ} \mathrm{C}$. The temperature range satisfactory for the enhanced secondary metabolites production is usually narrow, for example, $5-10^{\circ} \mathrm{C}$ (Iwai and Omura, 1982). Incubation period up to 7 days was found to be significant for antimicrobial compound production by $P$. elgii and highest production was recorded at third day. These results clearly indicated that various environmental factors and cultural conditions like incubation temperature, $\mathrm{pH}$ and incubation period were found to have a thoughtful 
impact on antimicrobial compound production by P. elgii. In our study also TSB supplemented with glucose and meat peptone with $\mathrm{pH} 7$ and incubated at $30^{\circ} \mathrm{C}$ for 3 days were the optimal condition for antimicrobial metabolite production by $P$. elgii.

\section{CONCLUSION}

In the present study, $P$. elgii isolated from the forest soil of Wayanad, Kerala, India recorded prominent broad spectrum activity against many medically important microbial pathogens. TSB supplemented with glucose and meat peptone with $\mathrm{pH} 7$ and

\section{REFERENCES}

Ajesh, K., Sudarslal, S., Arunan, C., and Sreejith, K. (2013). Kannurin, a novel lipopeptide from Bacillus cereus strain AK1: isolation, structural evaluation and antifungal activities. J. Appl. Microbiol. 115, 1287-1296. doi: 10.1111/jam.12324

Ash, C., Priest, F. G., and Collins, M. D. (1993). Molecular identification of rRNA group 3 bacilli (Ash, Farrow, Wallbanks and Collins) using a PCR probe test. Antonie Van Leeuwenhoek 64, 253-260. doi: 10.1007/BF00873085

Blunt, J. W., Copp, B. R., Hu, W. P., Munro, M. H. G., Northcote, P. T., and Prinsep, M. R. (2009). Marine natural products. Nat. Prod. Rep. 26, 170-244. doi: $10.1039 / \mathrm{b} 805113 \mathrm{p}$

Calvo, A. M., Wilson, R. A., Bok, J. W., and Keller, N. P. (2002). Relationship between secondary metabolism and fungal development. Microbiol. Mol. Biol. Rev. 66, 447-459. doi: 10.1128/MMBR.66.3.447-459.2002

Demain, A. L. (1999). Pharmaceutically active secondary metabolites of microorganisms. Appl. Microbiol. Biotechnol. 52, 455-463. doi: $10.1007 / \mathrm{s} 002530051546$

Drummond, A. J., Ashton, B., Buxton, S., Cheung, M., Cooper, A., Duran, C. et al. (2012). Geneious, v5.6. Available http://www.geneious.com/ [accessed 12 January, 2012].

Elo, S., Suominen, I., Kampfer, P., Juhanoja, J., Salkinoja- Salonen, M., and Haahtela, K. (2001). Paenibacillus borealis sp. nov., a nitrogen-fixing species isolated from spruce forest humus in Finland. Int. J. Syst. Evol. Microbiol. 51, 535-545. doi: 10.1099/00207713-51-2-535

Feng, J., Zhang, W., Han, L., and Zhang, X. (2011). Statistical optimization of medium components to improve the antibiotic activity of Streptomyces sp. 19G-317. Afr. J. Agric. Res. 6, 4424-443

Gesheva, V., Ivanova, V., and Gesheva, R. (2005). Effects of nutrients on the production of AK-111-81 macrolide antibiotic by Streptomyces hygroscopicus. Microbiol. Res. 160, 243-248. doi: 10.1016/j.micres.2004.06.005

Guimaraes, L. M., Furlan, R. L., Garrido, L. M., Ventura, A., Padilla, G., and Facciotti, M. C. (2004). Effect of $\mathrm{pH}$ on the production of the antitumor antibiotic retamycin by Streptomyces olindensis. Biotechnol. Appl. Biochem. 40, 107-111. doi: 10.1042/BA20030166

Gunnarsson, N., Bruheim, P., and Nielsen, J. (2003). Production of the glycopeptide antibiotic A40926 by Nonomuraea sp. ATCC 39727: influence of medium composition in batch fermentation. J. Ind. Microbiol. Biotechnol. 30, $150-156$.

Hassan, M. A., El-Naggar, M. Y., and Said, W. Y. (2001). Physiological factors affecting the production of an antimicrobial substance by Streptomyces violatus in batch cultures. Egypt. J. Biol. 3, 1-10.

Iwai, Y., and Omura, S. (1982). Cultural conditions for screening of new antibiotics. J. Antibiot. 35, 123-141. doi: 10.7164/antibiotics.35.123

Jose, P. A., and Jebakumar, S. R. D. (2013). Non-streptomycete actinomycetes nourish the current microbial antibiotic drug discovery. Front. Microbial. 4:240. doi: $10.3389 /$ fmicb. 2013.00240

Jose, P. A., Santhi, V. S., and Jebakumar, S. R. D. (2011). Phylogenetic- affiliation, antimicrobial potential and PKS gene sequence analysis of moderately halophilic Streptomyces sp. inhabiting an Indian saltpan. J. Basic Microbiol. 51, 348-356. doi: 10.1002/jobm.201000253

Kumar, S. N., Nambisan, B., Ramya, R., and Mohandas, C. (2013). Influence of carbon and nitrogen sources on antifungal metabolite production incubated at $30^{\circ} \mathrm{C}$ for 3 days were the optimal condition for the enhanced antimicrobial metabolite production by $P$. elgii.

\section{ACKNOWLEDGMENTS}

We thank Director CSIR-NIIST for his kind permission to carry out and publish this work. Dr. SK thanks, Kerala State Council for Science, Technology and Engineering (KSCSTE) for PDF fellowship. Acknowledgment to Department of Science and Technology (DST) for providing INSPIRE fellowship to JJ (IF 130648).

by bacterium associated with entomopathogenic nematode against Penicillium expansum. Arch. Phytopathol. Plant Protect. 46, 721-731. doi: 10.1080/03235408.2012.750815

Kumar, S. N., Siji, J. V., Ramya, R., Nambisan, B, and Mohandas, C. (2012). Improvement of antimicrobial activity of compounds produced by Bacillus sp. associated with a Rhabditid sp. (Entonopathogenic nematode) by changing carbon and nitrogen sources in fermentation media. J. Microbiol. Biotechnol. Food Sci. 1, 1424-1438.

Li, J., Beatty, P. K., Shah, S., and Jensen, S. E. (2007). Use of PCR-targeted mutagenesis to disrupt production of fusarioidin-type antifungal antibiotics in Paenibacillus polymyxa. Appl. Environ. Microbiol. 73, 3480-3489. doi: 10.1128/AEM-02

Mitchell, K. F., Taff H. T., Cuevas, M. A., Reinicke, E. L., Sanchez, H., and Andesa, D. R. 2013). Role of matrix $\beta-1,3$ glucan in antifungal resistance of nonalbicans Candida biofilms. Antimicrob. Agents Chemother. 57, 1918-1920. doi: 10.1128/AAC.02378-12

Naing, K. W., Anees, M., Kim, S. J., Nam, Y., Kim, Y. C., and Kim, K. Y. (2014). Characterization of antifungal activity of Paenibacillus ehimensis KWN38 gainst soil berne phytopathogenic fungi belonging to various taxonomic groups.Ann. Microbiol. 64, 55-63. doi: 10.1007/s13213-013-0632-y

Narayana, K. J. P., and Vijayalakshmi, M. (2008). Optimization of antimicrobial metabolites production by Streptomyces albidoflavus. Res. J. Pharmacol. 2, 4-7. guyen, T., Ishida, K., Jenke-Kodama, H., Dittmann, E., Gurgui, C., and Hochmuth, T. (2008). Exploiting the mosaic structure of trans-acyltransferase polyketide synthases for natural product discovery and pathway dissection. Nat. Biotechnol. 26, 225-233. doi: 10.1038/nbt1379

Pandey, A., Shukla, A., and Majumdar, S. K. (2005). Utilization of carbon and nitrogen sources by Streptomyces kanamyceticus M 27 for the production of an antibacterial antibiotic. Afr. J. Biotechnol. 4, 909-910.

Ridley, C. P., Lee, H. Y., and Khosla, C. (2008). Evolution of polyketide synthases in bacteria. Proc. Natl. Acad. Sci. U.S.A. 105, 4595-4600. doi: 10.1073/pnas.0710107105

Rodriguez-Diaz, M., Lebbe, L., Rodelas, B., Heyrman, J., De Vos, P., and Logan, N. A. (2005). Paenibacillus wynnii sp. nov., a novel species harbouring the nifH gene, isolated from Alexander Island, Antarctica. Int. J. Syst. Evol. Microbiol. 55, 2093-2099. doi: 10.1099/ijs.0.63395-0

Roux, V., Fenner, L., and Raoult, D. (2008). Paenibacillus provencensis sp. nov., isolated from human cerebrospinal fluid, and Paenibacillus urinalis sp. nov., isolated from human urine. Int. J. Syst. Evol. Microbiol. 58, 682-687. doi: 10.1099/ijs.0.65228-0

Roy, A., Mahata, D., Paul, D., Korpole, S., Franco, O. L., and Mandal, S. M. (2013). Purification, biochemical characterization and self-assembled structure of a fengycin-like antifungal peptide from Bacillus thuringiensis strain SM1. Front. Microbiol. 4:332. doi: 10.3389/fmicb.2013.00332

Ryu, C. M., Kim, J., Choi, O., Kim, S. H., and Park, C. S. (2006). Improvement of biological control capacity of Paenibacillus polymyxa E681 by seed pelleting on sesame. Biol. Control 39, 282-289. doi: 10.1016/j.biocontrol.2006. 04.014

Serrano, N. F. G., Rodrigues, L., Hokka, C. O., Sousa, C. P., Teixeira, J. A., and Mussatto, S. I. (2012). Optimal glucose and inoculum concentrations for production of bioactive molecules by Paenibacillus polymyxa RNC-D. Chem. Papers 66, 1111-1117. doi: 10.2478/s11696-012-0242-3 
Sole, M., Francia, A., Rius, N., and Loren, J. G. (1997). The role of pH in the glucose effect on prodigiosin production by non-proliferating cells of Serratia marcescens. Lett. Appl. Microbiol. 25, 81-84. doi: 10.1046/j.1472765X.1997.00171.x

Vaara, M., Siikanen, O., Apajalahti, J., Fox, J., Frimodt-Møller, N., He, H., et al. (2010). A novel polymyxin derivative that lacks the fatty acid tail and carries only three positive charges has strong synergism with agents excluded by the intact outer membrane. Antimicrob. Agents Chemother. 54, 3341-3346. doi: 10.1128/AAC.01439-09

Velkov, T., Thompson, P. E., Nation, R. L., and Li, J. (2010). Structure-activity relationships of polymyxin antibiotics. J. Med. Chem. 53, 1898-1916. doi: 10.1021/jm900999h

Vijayakumari, S. J., Sasidharannair, N. K., Nambisan, B., and Mohandas, C. (2013). Optimization of media and temperature for enhanced antimicrobial production by bacteria associated with Rhabditis sp. Iran. J. Microbiol. 5, 136-141.

Vilches, C., Mendez, C., Hardisson, C., and Salas, J. A. (1990). Biosynthesis of oleandomycin by Streptomyces antibioticus: influence of nutritional conditions and development of resistance. J. Gen. Microbiol. 136, 1447-1454. doi: 10.1099/00221287-136-8-1447

Wang, Y., Fang, X., An, F., Wang, G., and Zhang, X. (2011). Improvement of antibiotic activity of Xenorhabdus bovienii by medium optimization using response surface methodology. Microb. Cell Fact. 10, 98. doi: 10.1186/14752859-10-98

Wang, Y. H., Feng, J. T., Zhang, Q., and Zhang, X. (2008). Optimization of fermentation condition for antibiotic production by Xenorhabdus nematophila with response surface methodology. J. Appl. Microbiol. 104, 735-744. doi: 10.1111/j.1365-2672.2007.03599.x

Wright, G. D., and Sutherland, A. D. (2007). New strategies for combating multidrug-resistant bacteria. Trends Mol. Med. 13, 260-267. doi: 10.1016/j.molmed.2007.04.004

Zhang, H. W., Song, Y. C., and Tan, R. X. (2006). Biology and chemistry of endophytes. Nat. Prod. Rep. 23, 753-771. doi: 10.1039/b609472b

Conflict of Interest Statement: The authors declare that the research was conducted in the absence of any commercial or financial relationships that could be construed as a potential conflict of interest.

Copyright (c) 2015 Kumar, Jacob, Reshma, Rajesh and Kumar. This is an open-access article distributed under the terms of the Creative Commons Attribution License (CC BY). The use, distribution or reproduction in other forums is permitted, provided the original author(s) or licensor are credited and that the original publication in this journal is cited, in accordance with accepted academic practice. No use, distribution or reproduction is permitted which does not comply with these terms. 